\title{
Arabidopsis thaliana Nudix hydrolase AtNUDT7 forms complexes with the regulatory RACK1A protein and Ggamma subunits of the signal transducing heterotrimeric $\mathrm{G}$ protein
}

\author{
Kamil Olejnik\#, Maria Bucholc, Anna Anielska-Mazur, Agata Lipko, Martyna Kujawa, Marta \\ Modzelan, Agnieszka Augustyn* and Elzbieta Kraszewska ${ }^{\star}$
}

Institute of Biochemistry and Biophysics, Polish Academy of Sciences, Warszawa, Poland

\begin{abstract}
Arabidopsis thaliana AtNUDT7 Nudix pyrophosphatase hydrolyzes NADH and ADP-ribose in vitro and is an important factor in the cellular response to diverse biotic and abiotic stresses. Several studies have shown that loss-of-function Atnudt7 mutant plants display many profound phenotypes. However the molecular mechanism of AtNUDT7 function remains elusive. To gain a better understanding of this hydrolase cellular role, proteins interacting with AtNUDT7 were identified. Using AtNUDT7 as a bait in an in vitro binding assay of proteins derived from cultured Arabidopsis cell extracts we identified the regulatory protein RACK1A as an AtNUDT7-interactor. RACK1A-AtNUDT7 interaction was confirmed in a yeast two-hybrid assay and in a pull-down assay and in Bimolecular Fluorescence Complementation (BiFC) analysis of the proteins transiently expressed in Arabidopsis protoplasts. However, no influence of RACK1A on AtNUDT7 hydrolase catalytic activity was observed. In vitro interaction between RACK1A and the AGG1 and AGG2 gamma subunits of the signal transducing heterotrimeric $\mathrm{G}$ protein was also detected and confirmed in BiFC assays. Moreover, association between AtNUDT7 and both AGG1 and AGG2 subunits was observed in Arabidopsis protoplasts, although binding of these proteins could not be detected in vitro. Based on the observed interactions we conclude that the AtNUDT7 Nudix hydrolase forms complexes in vitro and in vivo with regulatory proteins involved in signal transduction. Moreover, we provide the initial evidence that both signal transducing gamma subunits bind the regulatory RACK1A protein.
\end{abstract}

Keywords: Arabidopsis thaliana, Nudix, AtNUDT7, RACK1A, heterotrimeric $\mathrm{G}$ protein, protein complexes

Received: 14 June, 2011; revised: 14 October, 2011; accepted: 25 October, 2011; available on-line: 08 November, 2001

\section{INTRODUCTION}

The AtNUDT7 (AtNUDX7, GFG1) pyrophosphatase belongs to the widely distributed Nudix protein family, characterized by the presence of a highly conserved Nudix motif $\mathrm{GX}_{5} \mathrm{Ex}_{7} \mathrm{REUXEEXGU}$ (where $\mathrm{U}$ is usually Ile, Leu or $\mathrm{Val}$ ). Enzymes of this family catalyze the hydrolysis of a variety of nucleoside diphosphate derivatives and a role for Nudix hydrolases has been proposed in the control of cellular levels of these compounds (Bessman et al.,1996).
Twenty nine Nudix hydrolases have been identified in Arabidopsis thaliana, (Kraszewska, 2008) and one member of this family, AtNUDT7, has been extensively studied. In vitro analyses indicated that the AtNUDT7 enzyme preferentially hydrolyzes ADP-ribose and NADH (Olejnik et al., 2005; Ogawa et al., 2005). AtNUDT7 was also found to accumulate as a homodimer and correct dimer formation is important for its function (Olejnik et al., 2009).

AtNUDT7 expression increases rapidly in response to an avirulent bacterial pathogen and abiotic stressors (Jambunathan \& Mahalingam, 2006). Also, loss of function Atnudt7 mutant plants display dwarfism, curled leaves, microscopic cell death, constitutive expression of pathogenesis-related $(P R)$ genes, increased resistance to bacterial pathogens and raised levels of reactive oxygen species (ROS) and NADH (Jambunathan \& Mahalingam, 2006; Bartsch et al., 2006). These phenotypes, coupled with hyper-sensitivity to photooxidative stress, are consistent with AtNUDT7 being important for maintaining cellular homeostasis during ROS-induced stress by hydrolyzing excess NADH which is a potential source of superoxide (Jambunathan \& Mahalingam, 2006). Genetic epistasis studies established that growth inhibition, enhanced basal resistance to bacterial pathogens, accumulation of salicylic acid (SA) and single cell death in Atnudt7 mutants depend on a functional Enhanced Disease Susceptibility1 (EDS1) gene which is a central coordinator of plant defense and cell death programmes. It was proposed that AtNUDT7 helps to absorb potentially dangerous ROS which otherwise lead to EDS1-induced defense responses (Bartsch et al., 2006).

Other studies have revealed that the morphological phenotype of Atnudt7 mutant plants depends on the growth environment and is caused by the mutant's super-sensitivity to some oxidative stress stimuli (Ge et al., 2007). Measurements of SA accumulation in Atnudt7 plants under different conditions, accompanied by genetic analyses, revealed that AtNUDT7 modulates two distinct branches of the defense pathway that are independent of and dependent on SA accumulation, respectively. No significant changes in the cel-

e-mail: elzbietak@ibb.waw.pl

"Current address: Bio-Rad, Warszawa, Poland

Current address: Biochemistry Department, Warsaw Medical University, Warszawa, Poland

Abbreviations: DTT, ditiothreitol; GSH, gluthatione, reduced form; GSSG, gluthatione, oxidized form; NBT/BCIP, 5-bromo-4-chloro-3indolyl sulphate/nitroblue tetrazolium; PMSF, phenylmethanesulfonyl fluoride; SDS, sodium dodecyl sulphate. 
lular level of ADP-ribose, the preferred in vitro substrate of AtNUDT7, was observed in pathogen-infected or uninfected Atnudt7 mutant plants. However, several hours after infection increased level of $\mathrm{NADH}$, the second preferred substrate of the hydrolase, in the pathogen-challenged nudt7 mutant plants was seen. In addition, it was observed that the Atnudt7 mutation caused perturbation in the cellular antioxidant status measured by the GSH to GSSG ratio. From this finding, it was concluded that the alteration in cellular redox homeostasis caused by the Atnudt7 mutation primes the cells for the amplified defense response (Ge et al., 2007; 2008).

Contrary to the observations of Ge and coworkers, no changes in the levels of GSH and GSSG in the Atnudt7 mutant plants or plants overexpressing the AtNUDT7 protein under normal or oxidative stress conditions were seen by Ishikawa et al. (2009). Instead, it was claimed that under these conditions, the levels of NADH and ADPribose were higher in the Atnudt7 mutants and lower in plants overexpressing the hydrolase, compared to wildtype plants. Plants overexpressing AtNUDT7 and the Atnudt7 mutants also displayed enhanced tolerance and sensitivity to oxidative stress, respectively. However, a more recent study found no decrease in the NADH level nor differences in resistance to pathogens in plants overexpressing AtNUDT7 compared to the wild type (Jambunathan et al., 2010). Furthermore, a diminished hypersensitive response (HR), which is an additional defense-associated phenotype, was seen in pathogen-challenged Atnudt7 plants (Adams-Philips et al., 2008).

The contradiction in the reported phenotype of the Atnudt7 mutant has yet to be resolved and the precise molecular mechanism by which the AtNUDT7 hydrolase regulates the cellular response to stressful conditions remains elusive. One route to understanding the role of AtNUDT7 in this process is the identification of interacting partners. In this study, we used AtNUDT7 as a bait in an in vitro binding assay to search for protein partners of this Nudix enzyme. A regulatory protein RACK1A was identified as an AtNUDT7-interacting protein. The specificity of this interaction was verified in yeast and in vitro, although the presence of RACK1A had no influence on the enzymatic activity of the AtNUDT7 hydrolase in vitro. The interaction between AtNUDT7 and RACK1A was confirmed in Arabidopsis protoplasts by employing the Bimolecular Fluorescence Complementation (BiFC) assay. To further examine the mechanism of AtNUDT7 function in a plant cell we tested whether its partner RACK1A can bind components of the signal transducing heterotrimeric $G$ complex. The heterotrimeric guanine-nucleotide-binding $(G)$ proteins are composed of $\alpha-\beta$-, and $\gamma$-subunits, which in Arabidopsis are named GPA1, AGB1 and AGG1/AGG2, respectively. Upon activation by membrane-bound $G$ protein-coupled receptors, the subunits transmit signals to their downstream effectors. We established that RACK1A interacts with the AGG1 and AGG2 subunits of $G$ protein in vitro and in yeast. Interactions between RACK1A and both $G \gamma$ subunits and between AtNUDT7 and these subunits were also observed in Arabidopsis protoplasts. These data suggest that AtNUDT7 functions as part of a complex of regulatory proteins.

\section{MATERIAL AND METHODS}

Plant material and cDNA clones. The T87 Arabidopsis thatiana cell line (Axelos et al., 1992) was obtained from the RIKEN Plant Cell Bank (Tsubaka, Japan). Suspension cultures were grown in Gamborg B5 medium under continuous white light at $21^{\circ} \mathrm{C}$ on a rotary shaker, as described by Yamada et al. (2004). Weekly subculture of the cell line was performed by inoculating $50 \mathrm{ml}$ of fresh GB5 medium with a $5 \mathrm{ml}$ aliquot of saturated culture.

cDNA clones encoding AtNUDT7, RACK1A, GPA1, AGB1, AGG1 and AGG2 proteins were obtained from the Arabidopsis Biological Resource Center (Ohio, USA). The cDNAs were PCR-amplified and introduced in-frame into appropriate vectors. All DNA inserts were verified by sequencing before constructs were used in experiments. The oligonucleotide primers used in this study are listed in Supplementary Table 1 (at www.actabp.pl). In constructs encoding proteins expressed as $\mathrm{N}$-terminal fusions with tag polypeptides, the ATG start codons were omitted.

Preparation of a soluble protein extract from cultured Arabidopsis T87 cells. Arabidopsis T87 cells were collected by centrifugation and resuspended in phosphate-buffered saline (PBS) $\mathrm{pH}$ 7.4, supplemented with $10 \mathrm{mM}$ 2-mercaptoethanol, $50 \mathrm{mM}$ $\beta$-glycerophosphate, $100 \mu \mathrm{M} \mathrm{Na}_{3} \mathrm{VO}_{4}, 500 \mu \mathrm{M}$ PMSF, 1 $\mu \mathrm{M}$ pepstatin, $1 \mu \mathrm{M}$ leupeptin and $1 \mu \mathrm{M}$ aprotinin. The cells were then disrupted by sonication $(5 \times 30 \mathrm{~s})$ using a Sonifier 250 (Branson Ultrasonic Corp., Danbury, CT, USA). The cell lysate was cleared by centrifugation and the protein-containing supernatant was used for in vitro assays.

Expression and purification of recombinant proteins. The expression and purification of recombinant His-tagged-AtNUDT7 protein was performed essentially as described by Olejnik and Kraszewska (2005). For the production of glutathione $S$-transferase (GST)-tagged proteins, the cDNAs encoding either AtNUDT7 or RACK1A were cloned into expression vector pGEX4T-1 to produce fusions with a gene encoding GST. Recombinant GST-tagged proteins were expressed in Escherichia coli BL21(DE3) or Rosetta cells (Novagen) and purified as described in the GE Healthcare manual.

Hydrolase assay. The catalytic properties of the purified, His-tagged-AtNUDT7 were determined, in the presence or absence of RACK1A, using ADP-ribose or $\mathrm{NADH}$ as a substrate in a hydrolase assay, as described by Olejnik et al. (2009). Standard $25 \mu \mathrm{l}$ reaction mixtures were composed of $50 \mathrm{mM}$ Tris/ $\mathrm{HCl}, \mathrm{pH} 7.0,5 \mathrm{mM}$ $\mathrm{MgCl}_{2}, 1 \mathrm{mM}$ DT'T, ADP-ribose or NADH and 0.1-1.0 milliunits of freshly purified AtNUDT7. After incubation at $37^{\circ} \mathrm{C}$ for $30 \mathrm{~min}$ the reactions were stopped by addition of $1 \mu \mathrm{l}$ of $20 \mathrm{mM}$ EDTA, then 1 unit of alkaline phosphatase was added and the incubation continued for an additional $15 \mathrm{~min}$ at $37^{\circ} \mathrm{C}$. The reaction was terminated by addition of $275 \mu \mathrm{l}$ of $20 \mathrm{mM}$ EDTA and the resulting inorganic orthophosphate content was determined colorimetrically as described by Ames and Dubin (1960). Under optimal conditions, one unit of enzyme catalyzed the hydrolysis of $1 \mu \mathrm{mol}$ of substrate per minute. In some cases, AtNUDT7 in $50 \mathrm{mM}$ Tris/ $\mathrm{HCl}, \mathrm{pH} \mathrm{7.0,} \mathrm{and} 1 \mathrm{mM}$ DTT was incubated with an equal amount of the RACK1A protein for $15 \mathrm{~min}$ on ice, prior to the hydrolase assay.

GST pull-down assays. For GST pull-down assays, $100 \mu \mathrm{l}$ of S-Hexylglutathione agarose beads carrying bound recombinant GST-tagged AtNUDT7 or GST-tagged RACK1A proteins $(2 \mu \mathrm{g} / \mu \mathrm{l})$ were used as the bait. Beads with GST alone at the same concentration served as a control. The beads carrying proteins 
were incubated with either $3 \mathrm{ml}$ of the $A$. thaliana T87 cell protein extract (protein concentration $3 \mu \mathrm{g} / \mu \mathrm{l}$ ) or $1 \mathrm{ml}$ of extracts of $E$. coli cells expressing His-tagged AGG1 or AGG2 proteins (protein concentration $3 \mu \mathrm{g}$ / $\mu \mathrm{l})$, for $2 \mathrm{~h}$ at $4^{\circ} \mathrm{C}$ on a rotary shaker. The beads with bound protein complexes were washed extensively with PBS supplemented with $10 \mathrm{mM}$ 2-mercaptoethanol and $0.05 \%$ Tween 20 . The beads were then boiled with SDS gel-loading buffer to release protein complexes and these were separated by electrophoresis on $10 \% \mathrm{SDS} /$ polyacrylamide gels, followed by Coomassie Brilliant Blue staining and preparation for MS analysis, or electroblotted for Western analysis with either anti-His (Santa Cruz Biotechnology, USA) or anti-AtNUDT7 antibodies (BioGenes GmbH, Germany). Each experiment was repeated at least three times with similar results.

Electrospray ionization mass spectrometry (ESIMS). To identify potential partners of AtNUDT7, bands of proteins isolated during pull-down assays were excised from SDS/polyacrylamide gels, reduced, alkylated, digested overnight with trypsin and then analyzed by tandem mass spectrometry using a Q-Tof1 mass spectrometer (Micromass, UK) combined with nano-HPLC (LC Packings). The MaxEnt3 program (Micromass, UK) was used to identify the obtained peptide sequences.

Western analysis. Western analysis was performed essentially as described in the Invitrogen manual. Proteins were separated on $10 \%$ SDS/polyacrylamide gels and transferred to nitrocellulose membrane by electroblotting. The membrane was blocked for $1 \mathrm{~h}$ at room temperature in TBSB buffer $(10 \mathrm{mM}$ Tris/ $\mathrm{HCl}, \mathrm{pH} 7.5$, $100 \mathrm{mM} \mathrm{NaCl}, 2 \% \mathrm{BSA}$ ) and then incubated for $2 \mathrm{~h}$ in the same buffer containing 1:2000 primary anti-His-tag antibody (Santa Cruz Biotechnology, USA) or 1:2000 polyclonal anti-AtNUDT7 antibody (BioGenes $\mathrm{GmbH}$, Germany). Following extensive washing in TBST buffer (10 mM Tris/HCl, pH 7.5, $100 \mathrm{mM} \mathrm{NaCl,} \mathrm{0.1 \%} \mathrm{Tween}$ $20)$ to remove unbound antibody, the blots were incubated with an alkaline phosphatase-conjugated secondary antibody $(1: 1000)$ in TBSB buffer for $1 \mathrm{~h}$, then washed again in TBST and developed using the NBT/BCIP chromogenic substrate.

Yeast two-hybrid analysis. To study protein interactions yeast two-hybrid experiments were performed according to the Clontech Matchmaker Two-Hybrid user manual. cDNAs encoding AtNUDT7, GPA1, AGB1, AGG1 or AGG2 proteins were cloned into the pGBT9 (BD) vector. cDNAs encoding RACK1A or the AGG1 protein were cloned into the pGAD424 (AD) vector. $\mathrm{AD}$ and $\mathrm{BD}$ constructs were introduced into the yeast strain PJ69-4A by the lithium acetate method (James et al., 1996). The transformed yeast cells were initially grown on plates containing medium lacking tryptophan and leucine (-Trp/Leu). These strains were then streaked onto selective medium lacking either tryptophan, leucine and histidine (-HIS) or tryptophan, leucine and adenine $(-\mathrm{ADE})$. Interactions between the co-expressed proteins were monitored by assessing the growth of yeast transformants on selection media after $3-5$ days at $30^{\circ} \mathrm{C}$. For control experiments, yeast cells were co-transformed with a plasmid carrying cDNA encoding one of the proteins plus the appropriate empty vector (BD or $\mathrm{AD})$. Each experiment was repeated at least three times with similar results.

Protoplast preparation, transient expression assays and microscopic analysis. For protoplast transformation, cDNAs encoding the investigated proteins were cloned into pSAT vectors kindly provided by Dr.
Tzvi Tzifra (Tzifra et al., 2005). In order to visualize their location in cells, the AtNUDT7, RACK1A, AGG1 and AGG2 proteins were fused to the $C$ terminus of enhanced green fluorescent protein (EGFP; vector pSAT6-EGFP-C1). To visualize the interaction of two proteins the cDNAs were subcloned from pSAT6-EYFP-C1 constructs into either pSAT4-nEYFP-C1-B or pSAT4-cEYFP-C1-B. Appropriate pairs of the final constructs were introduced into Arabidopsis protoplasts.

Arabidopsis T87 protoplasts were isolated and transformed via PEG treatment in sterile conditions according to the protocol of $\mathrm{He}$ and coworkers (2007), with minor modifications. In particular, the saccharose gradient purification step was omitted. In each transformation, about $2 \times 10^{5}$ protoplasts were transfected with approx. $40 \mu \mathrm{g}$ of plasmid DNA; for the BiFC experiments the pairs of constructs were mixed in a $1: 1(\mathrm{w} / \mathrm{w})$ ratio. The transfected protoplasts were suspended in incubation solution (0.5 M mannitol, $4 \mathrm{mM}$ MES, pH 5.7, $20 \mathrm{mM} \mathrm{KCl}$ ) and incubated at $25^{\circ} \mathrm{C}$ in the dark for $34-40 \mathrm{~h}$.

For analysis, the protoplasts were carefully placed on slides within home-made chambers to prevent damage and drying. Fluorescent images were recorded using a Nikon EZ-C1 laser scanning microscope mounted on an inverted epifluorescence TE 2000E microscope (Nikon) equipped with a $60 \times($ NA 1.4) oil-immersion objective and Nomarski contrast (DIC). The $488 \mathrm{~nm}$ line from an argon-ion laser (40 mW, Melles Griot) was used for excitation of enhanced yellow fluorescent protein (EYFP). EYFP fluorescence was detected with a $535 / 30 \mathrm{~nm}$ band-pass filter and rendered in false green. DIC images were acquired using the transmission mode of the EZ-C1 confocal system. The recorded fluorescent images were $z$-series projections or single optical sections made with the standard EZ-C1 Nikon software. Images were processed with the EZ-C1 Viewer 3.6 and Adobe Photoshop 6.0 software. Each experimental variant was repeated at least 3 times with similar results.

\section{RESULTS}

\section{Identification of proteins interacting with AtNUDT7}

To identify potential cellular partners of AtNUDT7 we performed a pull-down assay using AtNUDT7 as a "bait" and a soluble protein extract from cultured Arabidopsis T87 cells as a source of interactors. For this experiment, the AtNUDT7 coding region was expressed as a glutathione $S$-transferase (GST) fusion in $E$. coli. The recombinant prostein was immobilized on glutathioneSepharose beads and incubated with the Arabidopsis protein extract. Beads carrying GST alone served as a

Table 1. Proteins identified as the potential partners of AtNUDT7.

Proteins from Arabidopsis cell extract that bound to AtNUDT7 in the pull-down assay were identified by mass spectrometry analysis.

\begin{tabular}{ll}
\hline Locus & Protein description \\
\hline At1g18080 & $\begin{array}{l}\text { RACK1A, WD-40 repeat protein, putative beta } \\
\text { subunit of G protein }\end{array}$ \\
At5g17920 & $\begin{array}{l}\text { AtMS1, cobalamin-independent methionine syn- } \\
\text { thase }\end{array}$ \\
At2g44060 & LEA, late embryogenesis abundant proteins \\
At1g20010 & TUB5, tubulin beta 5 chain \\
At5g23860 & TUB8, tubulin beta 8 chain \\
\hline
\end{tabular}




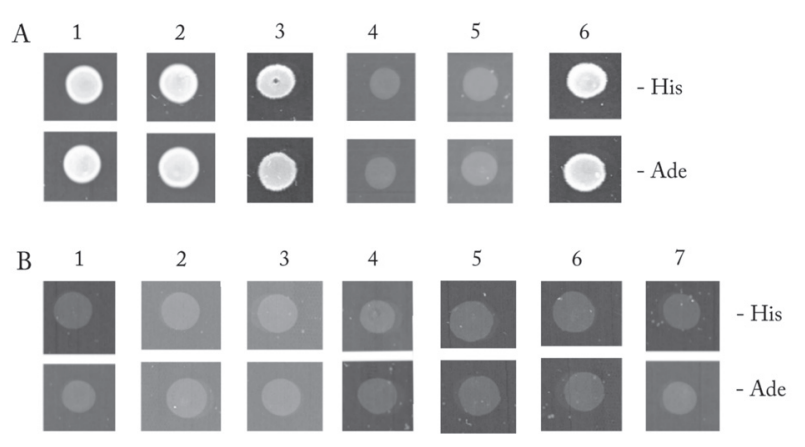

Figure 1. Yeast two-hybrid analysis of protein interactions. CDNAs encoding the RACK1A, AtNUDT7, GPA1, AGB1, AGG1 or AGG2 proteins were cloned into appropriate vectors and co-introduced into Saccharomyces cerevisiae strain PJ69-4A. Protein-protein interactions were monitored by growth of transformed yeast cells on selective media: (-HIS) or (-ADE). (A) Interactions between 1. AtNUDT7 and RACK1A, 2. RACK1A and AGG1, 3. RACK1A and $A G G 2,4$. RACK1A and $A G B 1,5$. RACK1A and GPA1, 6. AGB1 and AGG1. (B) Growth of yeast strains co-transformed with 1. AtNUDT7 and AGB1 or combinations of empty vector and constructs encoding one of the investigated proteins: 2. RACK1A, 3. AtNUDT7, 4. AGG1, 5. AGG2, 6. AGB1, 7. GPA1.

control. Following intense washing, bound proteins were separated on SDS/polyacrylamide gels. Protein bands were excised from the gels and analyzed by mass spectrometry. Several polypeptides that physically bind to AtNUDT7 were identified. The significant hits that were found consistently in three independent binding experiments and were not identified in the controls are listed in Table 1.

\section{AtNUDT7 interacts with the regulatory protein RACK1A}

The regulatory protein RACK1A was found to be among the interacting partners of AtNUDT7. Recently it was shown that in rice RACK1 functions in innate immunity by interacting with multiple proteins in the immune complex and plays a key role in ROS production (Shirasu et al., 2003; Nakashima et al., 2008). In Arabidopsis, RACK1A mediates hormonal responses, regulates multiple developmental processes and may be required for normal production of $60 \mathrm{~S}$ and $80 \mathrm{~S}$ ribosomes (Chen et al., 2006; Guo et al., 2011).

To confirm that RACK1A binds directly to AtNUDT7 we performed yeast two-hybrid analysis. The co-expression of the AtNUDT7 (BD) and RACK1A (AD) pro-

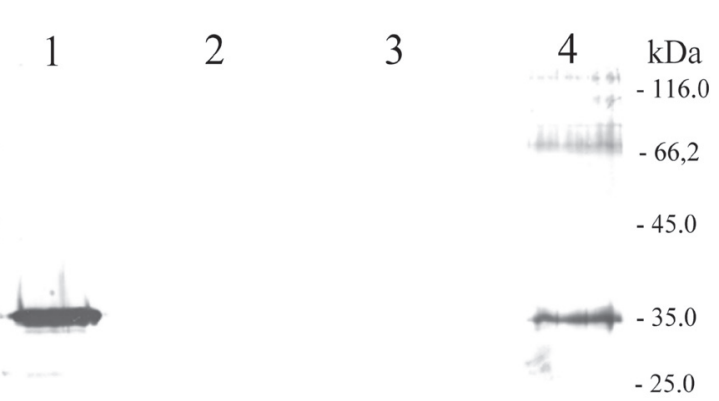

Figure 2. Interaction between RACK1A and the native form of AtNUDT7 examined with GST pull-down assays.

GST-RACK1A (bait) was immobilized on glutathione-Sepharose beads and incubated with Arabidopsis thaliana soluble protein extract. Following SDS/PAGE and electroblotting, AtNUDT7 was identified using anti-AtNUDT7 antibody. 1. Recombinant His ${ }_{6}$-AtNUDT7 protein; Arabidopsis proteins bound to 2. empty beads, 3 . beads carrying GST; 4 . beads carrying GST-RACK1A. The positions of the protein molecular mass marker bands are indicated. teins specifically activated His 3 and Ade2 reporter genes in the transformed yeast cells (Fig. 1A1). Separately, neither the AtNUDT7 nor RACK1A proteins were able to activate reporter gene transcription (Fig. 1B2 and B3).

The formation of complexes between these two proteins was further verified using a reciprocal pull-down assay. For these experiments, the RACK1A coding region was expressed as a GST fusion in E. coli. The recombinant protein was immobilized on glutathione-Sepharose beads and incubated with the Arabidopsis soluble protein extract. Beads carrying GST alone served as a control. Following several washes, bound proteins were separated on an SDS/polyacrylamide gel and analyzed by immunoblotting. The presence of the AtNUDT7 protein was detected with anti-AtNUDT7 antibody, confirming that RACK1A interacts with the native form of this $\mathrm{Nu}-$ dix hydrolase (Fig. 2, lane 4).

\section{RACK1A does not affect the catalytic activity of the AtNUDT7 hydrolase}

To determine whether RACK1A binding influences the catalytic properties of the AtNUDT7 hydrolase in vitro, its catalytic properties were estimated separately with ADP-ribose and NADH as the substrates in the presence and absence of the RACK1A partner (Table 2). The catalytic efficiency of AtNUDT7 in reactions with ADP-ribose and $\mathrm{NADH}$, measured by the $k_{\text {cat }} / K_{\mathrm{m}}$ ratio, was similar to that reported by Olejnik et al. (2009), with values of $2.3 \times 10^{3} \mathrm{M}^{-1} \cdot \mathrm{s}^{-1}$ and $2.8 \times 10^{3} \mathrm{M}^{-1} \cdot \mathrm{s}^{-1}$, respectively. In the presence of RACK1A the efficiency was not significantly altered, with $k_{\text {cat }} / K_{\mathrm{m}}$ values of $2.2 \times 10^{3}$ $\mathrm{M}^{-1} \cdot \mathrm{s}^{-1}$ and $2.6 \times 10^{3} \mathrm{M}^{-1} \cdot \mathrm{s}^{-1}$ for ADP-ribose and NADH, respectively. This result suggested that in vitro, RACK1A does not affect the catalytic properties of the AtNUDT7 hydrolase.

\section{RACK1A interacts with gamma subunits of the heterotrimeric G protein: AGG1 and AGG2}

To further characterize the function of AtNUDT7 in the plant cell, we investigated the interactions of its partner, RACK1A, with other cellular proteins.

The results of recent studies in yeast and mammals have indicated that RACK1, which structurally resembles the $\beta$ subunit of $G$ protein, can bind to subunits of the heterotrimeric guanine-nucleotide-binding $(G)$ protein and modulate their signaling activity (Dell et al., 2002; Chen et al., 2004; 2008). The heterotrimeric $G$ proteins are composed of $\alpha-, \beta$, and $\gamma$-subunits that, in Arabidopsis, are named GPA1, AGB1 and AGG1/ AGG2, respectively.

Genetic analyses have indicated that $G$ proteins mediate plant responses to bacterial and fungal pathogens, and abiotic stress (Perfus-Barbeoch et al., 2004). In addition, there is growing evidence that $G$ proteins mediate a variety of developmental processes and hormone signaling (Ding et al., 2008). Therefore, we tested whether the Arabidopsis RACK1A protein can interact with the GPA1, AGB1, AGG1 and AGG2 Arabidopsis G protein subunits in the yeast two-hybrid system. Specific activation of both His 3 and Ade2 reporter genes in transformed yeast cells was seen only with co-expression of RACK1A (AD) and AGG1 or AGG2 (BD) (Fig. 1, A2 and $A 3)$. No reporter gene activation was observed when RACK1A and AGB1 (Fig. 1, A4) or GPA1 (Fig. 1, A5) were expressed in the same cell. The well recognized interaction between AGB1 and AGG1 served as a posi- 
Table 2. Kinetic parameters of AtNUDT7 estimated in the absence and presence of RACK1A.

ADP-ribose or NADH at a concentration of 0.1 to $2 \mathrm{mM}$ were used for colorimetric measurements. The $K_{\mathrm{m}}$ and $V_{\max }$ were determined from nonlinear regression analysis. The $I_{\text {cat }}$ was calculated from $V_{\max }$ assuming one active site for monomer One unit of enzyme catalyzes the hydrolysis of $1 \mu \mathrm{mol}$ of substrate/min under optimal conditions.

\begin{tabular}{lcccccc}
\hline Protein & \multicolumn{1}{c}{$K_{\mathrm{m}} \mathrm{mM}$} & & $K_{\mathrm{cat}} \mathrm{s}^{-1}$ & \multicolumn{3}{c}{$k_{\mathrm{cat}} / K_{\mathrm{m}} \mathrm{M}^{-1} \cdot \mathrm{s}^{-1}$} \\
\hline & ADP-ribose & NADH & ADP-ribose & NADH & ADP-ribose & $\mathrm{NADH}$ \\
AtNUDT7 & $1.1 \pm 0.11$ & $1.2 \pm 0.11$ & 2.5 & 3.3 & $2.3 \times 10^{3}$ & $2.8 \times 10^{3}$ \\
AtNUDT7+RACK1A & $1.3 \pm 0.12$ & $1.3 \pm 0.12$ & 2.9 & 3.4 & $2.2 \times 10^{3}$ & $2.6 \times 10^{3}$ \\
\hline
\end{tabular}

Initially, the intracellular localization of the AtNUDT7 and RACK1A proteins was investigated. Constructs encoding AtNUDT7 and RACK1A fused at their N-termini to the enhanced green fluorescent protein (EGFP) were introduced into Arabidopsis protoplasts and fluorescence was detected tive control (Fig. 1, A6). Since RACK1A structurally resembles $\beta$ subunit of the $G$ protein, the interaction of AtNUDT7 with AGB1 was also tested. However, no transcription activation was observed in yeast cells coexpressing these proteins (Fig. 1, B1). Individually, neither the AGG1 nor the AGG2 proteins were able to activate transcription of the reporter genes (Fig. 1, B4 and B5). Similarly, no transcription activation was observed in cells expressing AGB1 or GPA1 alone (Fig. 1, B6 and B7). To verify the observation that RACK1A can form complexes with AGG1 and AGG2, we performed in vitro binding analysis using the pull-down assay. For this experiment the RACK1A coding region was expressed as a GST fusion in E. coli. The recombinant protein was immobilized on glutathione-Sepharose beads and incubated with extracts from E. coli cells expressing either AGG1 or AGG2, each fused to a six histidine peptide (His-tag). Beads carrying GST alone served as a control. Following several washes, bound proteins were separated on an SDS/polyacrylamide gel and analyzed by immunoblotting. The presence of the AGG1 and AGG2 proteins was detected with anti-His antibody, confirming that RACK1A interacts with both Arabidopsis Gy subunits in yeast and in vitro (Fig. $3 \mathrm{~A}$ lane 4 and $\mathrm{B}$ lane 4).

\section{Subcellular localization and interactions between the proteins in Arabidopsis protoplasts}

To test whether AtNUDT7 associates with RACK1A and whether RACK1A forms complexes with AGG1 and AGG2 in Arabidopsis cells, we analyzed in vivo protein interactions using the BiFC system and Arabidopsis protoplasts.
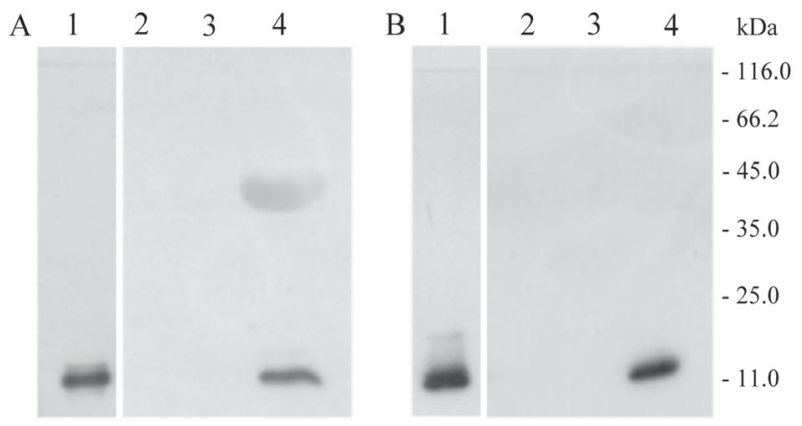

Figure 3. Interaction between RACK1A or AtNUDT7 with AGG1 and AGG2 examined with GST pull-down assays.

E. coli cell extracts containing His-tagged AGG1 or His-tagged AGG2 were incubated with glutathione-Sepharose beads carrying GST-RACK1A, GST-AtNUDT7 or GST alone. Following SDS/PAGE and electroblotting, the presence of the His-tagged proteins was identified using anti-His antibody. (A) Interactions with AGG1 protein and (B) interactions with AGG2 protein: 1. E. coli cell extract expressing either AGG1 or AGG2 (controls); proteins bound to 2 . beads carrying GST, 3. beads carrying GST-AtNUDT7, 4. beads carrying GST-RACK1A. The positions of the protein molecular mass marker bands are indicated. in the nucleus and the cytoplasm (Fig. 4A and B), suggesting that these proteins co-localize in the plant cell.
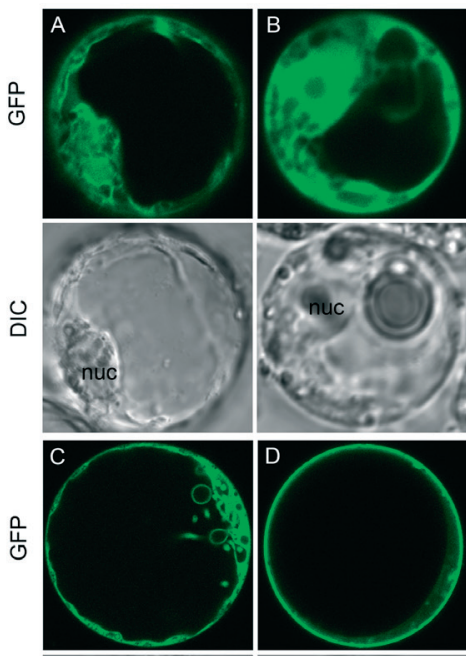

$\frac{0}{0}$
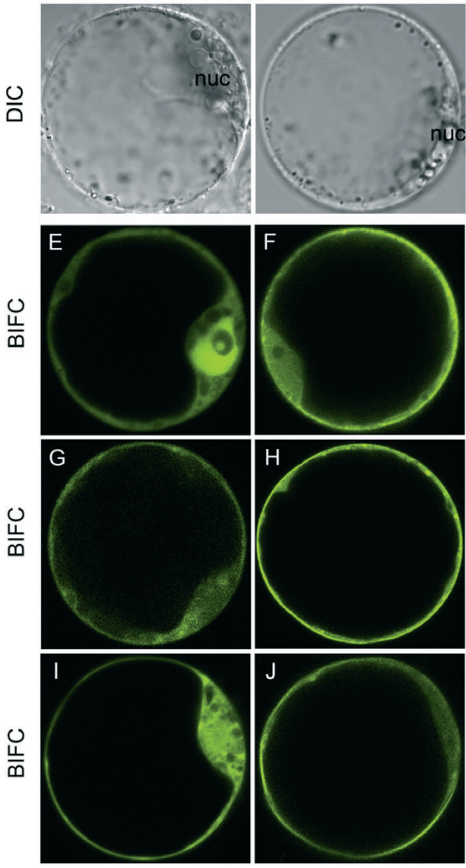

Figure 4. Subcellular localization of EGFP-protein fusions and in vivo interactions between the proteins detected by BiFC.

Localization of (A) EGFP-AtNUDT7, (B) EGFP-RACK1A, (C) EGFPCYFP-RACK1A, (F) nYFP-AGG2 and CYFP-RACK1A, (G) nYFP-AGG1 and CYFP-RACK1A, (H) nYFP-AGG2 and CYFP-AtNUDT7, (I) nYFPAGG1 and CYFP-AtNUDT7, (J) nYFP-AGG2 and CYFP-AGB1. DIC differential interference contrast (Nomarski contrast), nuc - nucleus. Representative images of Arabidopsis protoplasts transformed with the indicated constructs are presented. AGG1, (D) EGFP-AGG2. Interaction between (E) nYFP-AtNUDT7 and 
External stimuli (e.g. oxidative stress)

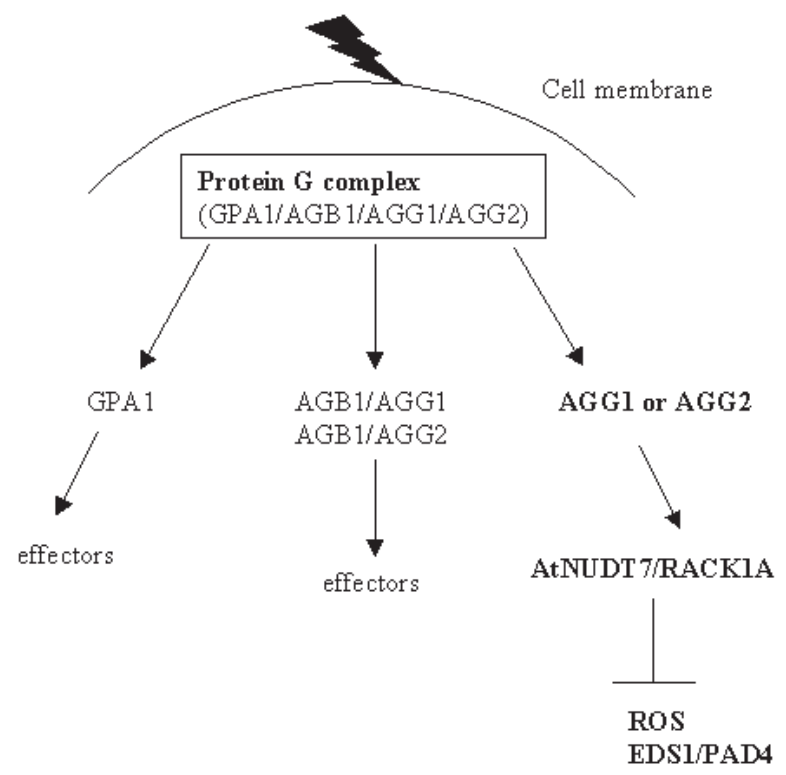

Figure 5. Graphical scheme illustrating the suggested functional links of AtNUDT7.

Upon external signals, transduced by specific receptors, the $G$ protein complexes dissociate into GPA1, AGB1/AGG1 or AGB1/ AGG2, AGG1 and $A G G 2$ subunits which transmit signals to their downstream effectors. Following binding of AGG1 or AGG2 (depending on the nature of a signal), AtNUDT7 (complexed with RACK1A) is activated and negatively regulates the cellular level of reactive oxygen (ROS) and a cellular defense pathway depend on a EDS1/PAD4 complex.

While the EGFP-AGG1 protein appeared dispersed in the cell, the EGFP-AGG2 protein was present predominantly at the plasma membrane (Fig. 4C and D). The observed localization of both Arabidopsis $G \gamma$ subunits is in agreement with that reported by Adjobo-Hermans et al. (2006). However, more peripheral localization of AGG1 was found by Wang and coworkers (2008).

Next we used BiFC to examine protein interactions in Arabidopsis protoplasts. Constructs expressing each of the investigated proteins as fusions with a complementary fragment of the enhanced yellow fluorescent protein (EYFP) were prepared. Appropriate fusion protein pairs were expressed in Arabidopsis protoplasts and an interaction between the proteins allowed the EYFP to adopt in its native three-dimensional structure and emit a fluorescent signal. The interaction between AtNUDT7 and RACK1A in both the nucleus and cytoplasm was confirmed (Fig. 4E). It has been shown previously that interaction with either $\mathrm{AGG} 1$ or $\mathrm{AGG} 2$ is required to relocate the AGB1 subunit to the cell periphery (Adjobo-Hermans et al., 2006; Wang et al., 2008).

However, we found that RACK1A was localized at the plasma membrane only when it associated with AGG2, whereas the RACK1A-AGG1 complex appeared more dispersed in the cell (Fig. 4F and G). Unexpectedly, we observed a fluorescence signal upon co-expression of EYFP fusions with AtNUDT7 and both the AGG1 and AGG2 subunits. Similarly to the RACK1A protein, the AtNUDT7 polypeptide moved to the cell periphery following binding to the AGG2 subunit, while the AtNUDT7-AGG1 complex was distributed between the nucleus and cytoplasm. (Fig. 4H and I). The known interaction between AGB1 and AGG2, observed mostly at the cell periphery by Wang et al. (2008), was used as a positive control for the BiFC analysis (Fig. 4J). No fluorescence signal was observed when protoplasts were transformed with the empty vectors, vectors encoding proteins which show no interaction (nYFP-RACK1A and cYFP-AGB1) or different combinations of the empty vectors and constructs encoding only one of the investigated proteins (Supplementary, Fig. 1 at www.actabp.pl).

To test whether AtNUDT7 binds G $\gamma$ in vitro, we used the pull-down assay. The recombinant GST-AtNUDT7 protein was immobilized on glutathione-Sepharose beads and incubated with the extract from E. coli cells expressing His-tagged versions of either AGG1 or AGG2. Beads carrying GST alone served as a control. Following several washes, bound proteins were separated on an SDS/polyacrylamide gel and analyzed by immunoblotting. Upon binding AtNUDT7, the presence of the $G \gamma$ subunits should be detected by anti-His antibody. However, no signal was observed, indicating that contrary to the situation with RACK1A, AtNUDT7 does not associate with AGG1 or AGG2 in vitro (Fig. 3A lane 3 and Fig. 3B lane 3). This result suggested that AtNUDT7 does not directly bind the $G \gamma$ subunits and additional protein(s) are required to mediate the interactions observed in the plant cell.

\section{DISCUSSION}

The results of this study indicate that the AtNUDT7 Nudix hydrolase may function as part of a complex of regulatory proteins. We have shown that AtNUDT7 interacts with RACK1A both in vitro and in vivo. Furthermore, in plant protoplasts, both RACK1A and AtNUDT7 associate with AGG1 and AGG2, the gamma subunits of the heterotrimeric $G$ protein signaling complex.

The RACK1 protein, originally identified as a receptor for activated protein kinase $\mathrm{C} 1$ in mammalian cells (Mocly-Rosen et al., 1991), was shown to serve as an adaptor protein for kinases, phosphatases, transcription factors and membrane receptors in a variety of mammalian signaling pathways. The sequence and structure of RACK1 proteins are highly conserved in eukaryotes (Sklan et al., 2006). In Arabidopsis, RACK1A mediates hormone responses and plays a regulatory role in multiple developmental processes. Loss-of-function mutations in rack $1 A$ confer defects in a variety of developmental processes including seed germination, leaf production and flowering (Chen et al., 2006; Guo et al., 2009). In addition, it was shown that RACK1A positively regulates ROS production and contributes to defense gene expression in rice cell cultures. Physical interactions between RACK1A and some key regulators of plant disease resistance including NADPH oxidase, RAR1 and SGT1 have also been observed (Nakashima et al., 2008). Furthermore, RACK1A was identified as one of seven proteins whose expression is down-regulated in the rice dwarf1 mutant lacking the $G \alpha$ subunit of the $G$ protein signaling complex (Komatsu et al., 2005). Thus, it was suggested that the function of RACK1A in the innate immunity of rice may be partially mediated through heterotrimeric $G$ proteins (Nakashima et al., 2008). Our findings that RACK1A physically interacts with both $\mathrm{G} \gamma$ subunits and also binds to AtNUDT7, a protein involved in cellular defense responses, could support this hypothesis. However, the recent observation that RACK1A did 
not interact with the $G \alpha$ (GPA1) or the G $\beta$ (AGB1) subunits of the $G$ complex led to the suggestion that RACK1A functions independently of the heterotrimeric $G$ protein in Arabidopsis (Guo et al., 2009). It is noteworthy that direct interaction between RACK1A and the G $\gamma$ subunits (AGG1 and AGG2) was not investigated by the authors. Moreover, it was shown earlier that the human pathogenic fungus Cryptococcus neoformans RACK1 homologue, the Gib2 protein, interacts with its two $G \gamma$ subunits and forms complexes, similar to the conventional G $\beta \gamma$, that function in the cAMP signaling pathway (Palmer et al., 2006).

We found that AtNUDT7, unlike RACK1A, does not interact with the $G \gamma$ subunits in vitro and so the observed association between these proteins in plant protoplasts is most probably facilitated by an intermediary, e.g. RACK1A. Both AtNUDT7 and RACK1A are known to form homodimers (Olejnik et al., 2005; Nakashima et al., 2008) and they can each potentially interact with two or more different proteins by recognition of the same motif (Liu et al., 2007). It may be speculated that in Arabidopsis, RACK1A functions as a scaffolding protein for a complex that consists of AtNUDT7 and the components of the heterotrimeric $G$ protein. Other components of this hypothetical complex may be proteins involved in defense or other cellular systems whose identity has yet to be discovered. Recent findings support the existence of such a complex. It was shown that in Arabidopsis, the GPA1 and AGB1 subunits of the G protein heterotrimer are associated with large (about $700 \mathrm{kDa}$ ) membrane-bound complexes. While a significant fraction of GPA1 was also present at the membrane as the free monomer, the AGB1 subunits were identified exclusively in the large complexes, most probably accompanied by their tightly bound partners, the gamma subunits (Wang et al., 2008). In addition, it was shown that the plasma membrane-bound NADPH oxidase, stimulates the initiation of EDS-1-dependent cell death in Atnudt7 mutants, indicating that this process requires the ROS signal generated by the oxidase. Increased level of ROS was also observed in the Atnudt7 mutants (Straus et al., 2010). It may be postulated that the AtNUDT7 protein senses the ROS changes through the gamma subunits of the $G$ protein and in turn hydrolyzes excess NADH, the compound that can potentially act as a source of superoxide through the concerted action of NADH kinase and NADPH oxidase (Torres \& Dangl, 2005). The enzymatic activity of AtNUDT7 during this process would have to be tightly regulated as the ratio of NADH/NAD is an important factor in the cellular redox balance. Therefore, functioning as part of a regulatory complex might be a prerequisite for this Nudix hydrolase. The recent observation of Jambunathan et al. (2010) that overexpression of the AtNUDT7 protein did not affect the cellular level of NADH and our finding that RACK1A had no influence on the catalytic activity of AtNUDT7 in vitro, indicate that some additional cellular components may be required to modulate the activity of this enzyme in the plant cell. The suggested functional links of AtNUDT7 are presented graphically on Fig. 5.

It is worth mentioning that the hydrolytic activity of Nudix proteins may not always be physiologically relevant to their biological function. Instead, binding of nucleotide analogs to the Nudix domain can cause conformational changes in a Nudix protein that may in turn influence the protein's biological activity. Recently, it was observed that ADP-ribose suppresses the in vitro binding of a bacterial transcription factor NrtR to its DNA target. In the proposed mechanism of transcriptional regulation by NrtR, its Nudix domain is responsible for specific binding of ADP-ribose. This interferes with the ability of its DNA-binding domain to bind to the operator sites in the promotor regions of genes involved in NAD metabolism (Rodionow et al., 2008). We showed here that the AtNUDT7 protein may also be located in the nucleus, and it would be interesting to investigate its function in this cellular compartment. In addition, the possibility that AtNUDT7 modulates the poly(ADPribosyl)ation (PAR) reaction involved in DNA repair programs in response to oxidative and biotic stresses has already been suggested (Adams-Philips et al., 2008; Ishikawa et al., 2009).

Although a full understanding of how protein complexes containing AtNUDT7 are regulated and function in the cell requires further work, the findings of the present study provide some novel insights into the biological activity of this Nudix protein. In addition, the results expand our knowledge about Nudix hydrolases and point to their participation in cellular processes that require protein complexes rather than solitary enzymes. Moreover, we provide initial evidence that the RACK1A protein can form complexes with the gamma subunit of the signal transducing heterotrimeric $G$ protein. Since little is known about interacting proteins of G-protein signaling in plants our observation adds to that information.

\section{Acknowledgements}

We would like to thank Dr. Tzvi Tzifra from the Department of Molecular, Cellular and Developmental Biology, University of Michigan (USA) for the kind gift of the pSAT vectors. We would also like to thank Dr. Jane Parker from the Max Planck Institute for Plant Breeding Research (Germany) for her valuable comments on this work.

This study was supported by grant No NN303351635 from the Ministry of Science and Higher Education.

\section{REFERENCES}

Ames BN, Dubin DT (1960) The role of polyaminesin neutralization of bacteriophage deoxyribonucleic acids. J Biol Chem 235: 769-775.

Adams-Phillips L, Wan J, Tan X, Dunnin FM, Meyers BC, Michelmore RW, Bent AF (2008) Discovery of ADP-ribosylation and other plant defense pathway elements through expression profiling of four different Arabidopsis-Pseudomonas R-avr interactions. Mol Plant Microbe Interact 21: 646-657.

Adjobo-Hermans MJW, Goedhart J, Gadella Jr TWJ (2006) Plant G protein heterotrimers require dual lipidation motifs of $G \alpha$ and $G \gamma$ and do not dissociate upon activation. J Cell Sci 119: 5087-5097.

Axelos M, Curie C, Mazzolini L, Bardet C, Lescure B (1992) A proto$\mathrm{col}$ for transient gene expression in Arabidopsis thaliana protoplasts isolated from cell suspension cultures. Plant Physiol Biochem 30: 123128.

Bartsch M, Gobbato E, Bednarek P, Debey S, Schultze JL, Bautor J, Parker JE (2006) Salicylic acid-independent enhanced disease susceptibility1 signaling in Arabidopsis immunity and cell death is regulated by the monooxygenase FMO1 and the Nudix hydrolase NUDT7. Plant Cell 18: 1038-1051.

Bessman MJ, Frick DN, O'Handley SF (1996) The Mut'T proteins or "Nudix" hydrolases, a family of versatile, widely distributed, "housecleaning" enzymes. J Biol Chem 271: 25059-25062.

Chen J, Ullah H, Temple B, Liang J, Guo J, Alonso MJ, Ecker JR, Jones AM (2006) RACK1 mediates multiple hormone responsiveness and developmental processes in Arabidopsis. J Exp Bot 57: 2696-2708.

Chen S, Dell EJ, Lin F, Sai J, Hamm HE (2004) RACK1 regulates specific functions of Gbetagamma. J Biol Chem 279:17861-17868.

Chen S, Lin F, Shin ME, Wang F, Shen L, Hamm HE (2008) RACK1 regulates directional cell migration by acting on Gbetagamma at the 
interface with its effectors PLC beta and PI3K gamma. Mol Biol Cell 19: 3909-3922.

Dell EJ, Connor J, Chen S, Stebbins EG, Skiba NP, Mochly-Rosen D, Hamm HE (2002) The betagamma subunit of heterotrimeric G proteins interacts with RACK1 and two other WD repeat proteins. J Biol Chem 277: 49888-49895.

Ding L, Chen JG, Jones AM, Assman SM (2008) Heterotrimeric G protein-coupled signalling in higher plants. Annu Plant Rev 33: 3063.

Ge X, Li G-J, Wang S-B, Zhu H, Zhu T, Wang X, Xia Y (2007) AtNUDT7, a negative regulator of basal immunity in Arabidopsis, modulates two distinct defense response pathways and is involved in maintaining redox homeostasis. Plant Physiol 145: 204-215.

Ge X, Xia Y (2008) The role of AtNUDT7, a Nudix hydrolase, in the plant defence response. Plant Signaling Behavior 3: 119-120.

Guo J, Wang S, Valerius O, Hall H, Zeng Q, Li JF, Weston DJ, Ellis BE, Cheng JG (2011) Involvement of Arabidopsis RACK1 in protein translation and its regulation by abscisic acid. Plant Physiol 155: 370-383.

Guo J, Wang S, Wang J, Huang WD, Liang J, Chen JG (2009) Dissection of the relationship between RACK1A and heterotrimeric G proteins in Arabidopsis. Plant Cell Physiol 50: 1681-1694.

Guo J, Wang J, Xi L, Huang W-D, Liang J, Chen J-G (2009) RACK1A is a negative regulator of ABA responses in Arabidopsis. J Exp Bot 60: 3819-3833.

He P, Shan L, Sheen J (2007) The use of protoplasts to study innate immune responses. Methods Mol Biol 354: 1-9.

Ishikawa K, Ogawa T, Hirosue E, Nakayama Y, Harada K, Fukusaki E, Yoshimura K, Shigeoka S (2009) Modulation of the poly(ADPribosyl)ation reaction via Arabidopsis ADP-ribose/NADH pyrophosphohydrolase, AtNUDX7, is involved in the response to oxidative stress. Plant Physiol 151: 741-754.

Jambunathan N, Mahalingam R (2006) Analysis of Arabidopsis growth factor gene 1 (GFG1) encoding a nudix hydrolase during oxidative signaling. Planta 224: 1-11.

Jambunathan N, Penaganti A, Tang Y, Mahalingam R (2010) Modulation of redox homeostasis under suboptimal conditions by Arabidopsis nudix hydrolase 7. BMC Plant Biol 10:173 http://www. biomedcentral.com/1471-2229/10/173.

James P, Halladay J, Craig E A (1996) Genomic libraries and a host strain designed for highly efficient two-hybrid selection in yeast. Genetics 144: 1425-1436.

Komatsu S, Abbasi F, Kobasi E, Fujisawa Y, Kato H, Iwasaki Y (2005) Proteomic analysis of rice embryo: an approach for investigating G alpha protein-regulated proteins. Proteomics 5: 3932-3941.

Kraszewska E (2008) The plant Nudix hydrolase family. Acta Biochim Pol 55: 663-771.

Liu YV, Baek JH, Zhang H, Diez R, Cole RN, Semenza GL (2007) RACK1 competes with HSP90 for binding to HIF-1 $\alpha$ and is required for $\mathrm{O}_{2}$-independent and HSP90 inhibitor-induced degradation of HIF-1 $\alpha$. Mol Cell 25: 207-217.

Mochly-Rosen D, Khaner H, Lopez J (1991) Identification of intracellular receptor proteins for activated protein kinase C. Proc Natl Acad Sci USA 88: 3997-4000.
Nakashima A, Chen L, Thao NP, Fujiwara M, Wong HL, Kuwano M, Umemura K, Shirasu K, Kawasaki T, Shimamoto K (2008) RACK 1 functions in rice innate immunity by interacting with Rac1 immune complex. Plant Cell 20: 2265-2279.

Ogawa T, Ueda Y, Yoshimura K, Shigeoka S (2005) Comprehensive analysis of cytosolic Nudix hydrolases in Arabidopsis thaliana. J Biol Chem 280: 25277-25283.

Olejnik K, Kraszewska E (2005) Cloning and characterization of an Arabidopsis thaliana Nudix hydrolase homologous to the mammalian GFG protein. Biochim Biophys Acta 1752: 133-141.

Olejnik K, Plochocka D, Grynberg M, Goch G, Gruszecki WI, Basinska T, Kraszewska E (2009) Mutational analysis of the AtNUDT7 Nudix hydrolase from Arabidopsis thaliana reveals residues required for protein quarternary structure formation and activity. Acta Biochim Pol 56: 291-300.

Palmer DA, Thompson JK, Li L, Prat A, Wang P (2006) Gib2, a novel Gb-like/RACK1 homolog, functions as a Gb subunit in cAMP signaling and is essential in Cryptococcus neoformans. I Biol Chem 281: 32596-32605.

Perfus-Barbeoch L, Jones AM, Assman SM (2004) Plant heterotrimeric $G$ protein function: insights from Arabidopsis and rice mutants. Curr Opin Plant Biol 7: 719-731.

Rodionov DA, De Ingeniis J, Mancini C, Cimadamore F, Zhang $\mathrm{H}$, Osterman AL, Raffaelli N (2008) Transcriptional regulation of NAD metabolism in bacteria: NrtR family of Nudix-related regulators. Nucleic Acid Res 36: 2047-2059.

Shirasu K, Schulze-Lefert P (2003) Complex formation, promiscuity and multi-functionality: Protein interactions in disease-resistance pathways. Trends Plant Sci 8: 252-258.

Sklan EH, Podoly E, Soreq H (2006) RACK1 has the nerve to act: structure meets function in the nervous system. Prog Neurobiol 78: $117-134$.

Straus MR, Rietz S, Ver Loren van Themaat E, Bartsch M, Parker JE (2010) Salicylic acid antagonism of EDS1-driven cell death is important for immune and oxidative stress responses in Arabidopsis. Plant J 62: 628-640.

Torres MA, Dangl JL (2005) Functions of the respiratory burst oxidase in biotic interactions, abiotic stress and development. Curr Opin Plant Biol 8: 397-403.

Tzifra T, Tian G-W, Lacroix B, Vyas S, Li J, Leitner-Dagan Y, Krichevsky A, Taylor T, Citovsky V (2005) pSAT vectors: a modular series of plasmids for autofluorescent protein tagging and expression of multiple genes in plants. Plant Mol Biol 57: 503-516.

Wang S, Assmann SM, Fedoroff NV (2008) Characterization of the Arabidopsis heterotrimeric G protein. J Biol Chem 283: 13913-13922.

Yamada H, Koizumi N, Nakamichi N, Kiba T, Yamashino T, Mizuno $\mathrm{T}$ (2004) Rapid response of Arabidopsis T87 cultured cells to cytokinin through His-to-Asp phosphorelay signal transduction. Biosci Biotechnol Biochem 68: 1966-1976. 